\title{
ON THE ISOMORPHISMS BETWEEN CERTAIN CONGRUENCE GROUPS
}

\author{
ROBERT E. SOLAZZI
}

\begin{abstract}
In this paper we study the possible isomorphisms between the congruence subgroups of the classical groups over integral domains by applying the involution-free techniques previously used by O'Meara and the author. We prove, in dimensions at least 6 and characteristic not 2, that a linear congruence group is never isomorphic to a symplectic congruence group nor to an isotropic unitary congruence group whose associated hermitian space has Witt index at least 3 .
\end{abstract}

Introduction. Let $W$ be an $m$-dimensional vector space over the field $F, m \geqq 3$. Let - denote the natural homomorphism of $\mathrm{GL}(W)$ onto $\operatorname{PGL}(W)$. For any subgroup $S$ of $\mathrm{GL}(W)$, we define the projective group of $S$ (which we denote $P S$ ) to be the image of $S$ under the - homomorphism of $\mathrm{GL}(W)$ onto $\operatorname{PGL}(W)$.

Let $V$ be an $n$-dimensional vector space over a field $F_{1}$ of characteristic not 2 , and let $J$ be an involutionary automorphism of $F_{1}$. Let $(x, y)$ be a nondegenerate skew-hermitian form on $V$ with respect to $J$. That is

$$
(\lambda x+y, z)=\lambda(x, z)+(y, z) \text { and }(x, y)=-(y, x)^{J}
$$

for all $\lambda \in F_{1}, x, y, z \in V$. Define

$$
U_{n}(V)=\{\sigma \in \mathrm{GL}(V) \mid(\sigma x, \sigma y)=(x, y) \forall x, y \in V\} .
$$

Thus $U_{n}(V)$ is the n-dimensional symplectic group when $J$ is the identity map of $F_{1}$; otherwise $U_{n}(V)$ is a unitary group. We will assume $(x, y)$ has index at least 3 . For any subspace $U$ of $V$ we define the orthogonal complement $U^{*}$ of $U$ as $U^{*}=\{x \in V \mid(x, U)=0\}$. The radical of $U$, written $\operatorname{rad} U$, is defined as $\operatorname{rad} U=U \cap U^{*} . U$ is called regular if $\operatorname{rad} U=0, U$ is degenerate if $\operatorname{rad} U \neq 0$, and $U$ is totally degenerate if $\operatorname{rad} U=U$. Finally we say $U$ is isotropic if there is a nonzero vector $x$ in $U$ with $(x, x)=0$. A regular two-dimensional isotropic subspace of $V$ is called a hyperbolic plane.

Received by the editors June 20, 1971.

AMS 1970 subject classifications. Primary 20H05, 15A63; Secondary 15A57.

Key words and phrases. Classical group, centralizer, skew-hermitian form, linear, symplectic, unitary congruence group, transvection, isomorphism.

c. American Mathematical Society 1972 
Recall that an invertible linear transformation $\tau$ that fixes pointwise all vectors in some hyperplane is called a shearing; if det $\tau=1, \tau$ is a transvection. If det $\tau \neq 1, \tau$ is a dilation. If $\tau$ is a shearing and $\tau \neq 1$ then the image of $\tau-1$ is a line, called the proper line of $\tau$, and the hyperplane of fixed vectors of $\tau$ is called the proper hyperplane of $\tau$. Now we say a subgroup $G$ of $\operatorname{PGL}(W)$ is full of projective transvections if, for each line $L$ and hyperplane $H$ of $W$ with $L \subset H$, there is a nontrivial transvection $\tau$ in $\mathrm{GL}(W)$ with proper spaces $L \subset H$ such that $\bar{\tau} \in G$. Similarly we say a subgroup $G_{1}$ of $P U(V)$ has enough transvections if for each isotropic line $L$ of $V$ there is a nontrivial transvection $\tau$ in $U(V)$ with proper line $L$ such that $\bar{\tau} \in G_{1}$. In all that follows we will assume $G$ and $G_{1}$ are two subgroups of PGL( $W)$ and $P U(V)$ respectively such that $G$ is full of transvections and $G_{1}$ has enough transvections.

In all that follows whenever we refer to $G_{1}, G, V, W, F_{1}, F, n$ or $m$, we shall understand them to be defined as above. And we put:

$$
\Delta=\left\{\sigma \in \mathrm{GL}_{m}(W) \mid \bar{\sigma} \in G\right\}, \quad \Delta_{1}=\left\{\sigma \in U_{n}(V) \mid \bar{\sigma} \in G_{1}\right\} .
$$

If $\rho$ is a linear functional on $W$ and $a \in W$ we let $\tau_{a, \rho}$ denote the transvection defined by $\tau_{a, \rho}(x)=x+\rho(x) \cdot a$ for all $x \in W$. Similarly if $a$ is an isotropic vector in $V$ and $\lambda$ is an element of $F_{1}$ such that $\lambda=\lambda^{J}$ then $\tau_{a, \lambda}$ denotes the transvection in $U_{n}(V)$ defined by $\tau_{a, \lambda}(x)=x+\lambda(x, a) a$. If $X \subseteq \Delta$ or $X \subseteq \Delta_{1}, C(X)$ denotes the centralizer $C_{\Delta}(X)$ or $C_{\Delta_{1}}(X)$ respectively. If $X \subseteq G$ or $X \subseteq G_{1}, C(X)$ denotes the centralizer $C_{G}(X)$ or $C_{G_{1}}(X)$ respectively; and if $X$ is any group, $D X$ is the commutator subgroup. For a line $L$ and a hyperplane $H$ of $W$ with $L \subset H$ we let $\bar{T}(L, H)$ stand for the group of all projective transvections in $G$ having proper line $L$ and proper hyperplane $H$.

We say two elements $\sigma_{1}$ and $\sigma_{2}$ of $\operatorname{GL}(W)$ anticommute if $\bar{\sigma}_{1}$ and $\bar{\sigma}_{2}$ do commute, but $\sigma_{1}$ and $\sigma_{2}$ do not; i.e. $\sigma_{1} \sigma_{2}=\lambda \sigma_{2} \sigma_{1}$ for some scalar $\lambda$ in $F$ unequal to 0 or 1 . And if $a, b$ are any two elements of a group, $[a, b]$ denotes the commutator $a b a^{-1} b^{-1}$.

If $\sigma$ is an element of $\mathrm{GL}(W)$ we will call $(\sigma-1) W$ the residual space $R$ of $\sigma$. In what follows the letter $R$ will always denote the residual space of $\sigma$; similarly for $R_{i}$ and $\sigma_{i}$. And for a subspace $U$ of $W$ we define $E(U)=$ $\{\sigma \in \Delta \mid R \subseteq U\}$ where $R$ is the residual space of $\sigma$. Similarly if $U$ is a subspace of $V, E(U)$ will denote $\left\{\sigma \in \Delta_{1} \mid R \subseteq U\right\}$ where again $R$ is the residual space of $\sigma$.

1.1. Suppose that $\Sigma \in U_{n}(V)$ is such that $\Sigma\left(F_{1} a\right) \neq F_{1}$ a for some isotropic line $F_{1} a$ of $V$. Let $\tau_{a, \lambda}$ be a nontrivial transvection in $U_{n}(V)$. Then $\Sigma$ and $\tau_{a, \lambda} \Sigma^{-1} \tau_{a,-\lambda}$ do not commute. 
Proof. Suppose they did commute; then $\tau_{\Sigma a, \lambda} \tau_{a,-\lambda}=\tau_{a, \lambda} \tau_{\Sigma^{-1} a,-\lambda}$. Choose a vector $x$ orthogonal to $\Sigma^{-1} a$ but not to $a$. Upon substitution of $x$ in the above equation we obtain

$$
-\lambda(x, a) a+\lambda(x, \Sigma a) \Sigma a-\lambda^{2}(a, \Sigma a)(x, a) \Sigma a=\lambda(x, a) a
$$

which implies $a \in F \cdot \Sigma a$, a contradiction. Q.E.D.

1.2. Let $\sigma \in \Delta_{1}$ be such that $\operatorname{dim} R=2$ and $\sigma \mid R$ is not a scalar. Then $E(R) \subseteq C D C(\sigma)$.

Proof. As in 2.1 of [9]. Q.E.D.

1.3. Let $\sigma^{\star} \in \Delta_{1}$ and suppose $\operatorname{dim} R \leqq 2$. Then $C D C(\bar{\sigma}) \subseteq(E(R))^{-}$.

Proof. As in 2.2 of [9]. Q.E.D.

1.4. Let $\sigma \in \Delta_{1}$ be such that $R$ is a hyperbolic plane, and $\sigma \mid R$ is not a scalar. Then $C D C(\bar{\sigma})=(E(R))^{-}$.

Proof. Apply 1.2 and 1.3. Q.E.D.

1.5. Let $\sigma \in \Delta_{1}$ be such that $\operatorname{dim} R \leqq 2$. (a) If $R$ is totally isotropic, $C D C(\bar{\sigma})$ is abelian. (b) If $R$ is a nonisotropic line, $C D C(\bar{\sigma})$ is abelian. (c) If $R$ is a hyperbolic plane and $\sigma \mid R$ is not a scalar, then $C D C(\bar{\sigma})$ is nonabelian.

Proof. (a) follows from 1.3 and the fact if $\sigma_{1}$ and $\sigma_{2}$ are in $U_{n}(V)$ and $R_{1} \subseteq R_{2}^{*}$ then $\sigma_{1}$ and $\sigma_{2}$ commute.

(b) follows from 1.3.

To prove (c) observe that 1.2 implies $(E(R))^{-} \subseteq C D C(\bar{\sigma})$. Since $R$ is a hyperbolic plane there are two nonorthogonal isotropic lines $L_{1}$ and $L_{2}$ in $R$. If we choose two projective transvections in $G$ whose proper lines are $L_{1}$ and $L_{2}$, then these two projective transvections will be in $(E(R))^{-}$, hence in $C D C(\bar{\sigma})$, but will not commute. Q.E.D.

2.1. Let $W$ be an m-dimensional vector space over a field $F$ of any characteristic, $m \geqq 4$. Let $G$ be a subgroup of $\mathrm{PGL}_{m}(W)$ which is full of projective transvections and let $\bar{\sigma} \in G$ be a nontrivial projective transvection with proper spaces $L \subseteq H$. Then $C D C(\bar{\sigma}) \subseteq \bar{T}(L, H)$.

Proof. To begin, we claim that for any line $L_{1}$ in $H$ there is a nontrivial transvection $\tau$ in $D C(\sigma)$ with proper line $L_{1}$.

For let $L_{1}=F \cdot x_{1}$, let $L=F \cdot x_{0}$ and if $L_{1} \neq L$ extend $x_{0}$ and $x_{1}$ to a basis $x_{0}, x_{1}, \cdots, x_{m-1}$ for $W$ with $x_{0}, x_{1}, \cdots, x_{m-2}$ in $H$. Since $\Delta$ is full of transvections, there are nonzero $\alpha, \beta$ in $F$ such that $\tau_{x x_{1}, \rho_{2}}$ and $\tau_{x_{2}, \beta \rho_{3}}$ are in $\Delta$ where $\left(\rho_{i}\right)$ is the dual base of $\left(x_{i}\right)$. Clearly $\tau_{x x_{1}, \rho_{2}}$ and $\tau_{x_{2}, \beta \rho_{3}}$ are in $C(\sigma)$ by 
1.6 of [5], so $\tau=\left[\tau_{\alpha x_{1}, \rho_{2}} ; \tau_{x_{2}, \beta \rho_{3}}\right]=\tau_{\alpha x_{1}, \beta \rho_{3}}$ is a nontrivial transvection in $D C(\sigma)$ with proper line $L_{1}$.

Now if $L_{1}=L$ let $L_{1}=F \cdot x_{1}$ and extend $x_{1}$ to a base $x_{1}, \cdots, x_{m}$ for $W$ with $x_{1}, \cdots, x_{m-1}$ in $H$. Then reasoning as above we will obtain a nontrivial transvection in $D C(\sigma)$ with proper line $L_{1}$.

Now let $\bar{\Sigma} \in C D C(\bar{\sigma})$. Let $L_{1} \subseteq H$ and choose a nontrivial transvection $\tau$ in $D C(\sigma)$ with proper line $L_{1}$. Then $\bar{\tau} \in(D C(\sigma))^{-}=(D C(\sigma))^{-} \subseteq D C(\bar{\sigma})$. So $\bar{\Sigma}$ and $\bar{\tau}$ commute; $\Sigma$ and $\tau$ cannot anticommute since $\tau$ is a transvection and so $\Sigma$ and $\tau$ must commute. Hence $\Sigma L_{1}=L_{1}$ for all lines $L_{1}$ of $H$, and so $\Sigma \mid H=\alpha \cdot 1_{H}$ for some $\alpha \in \dot{F}$. Now let $\phi$ be any element of $D C(\sigma)$. Then $\bar{\phi} \in(D C(\sigma))^{-} \subseteq D C(\bar{\sigma})$. So $\bar{\phi}$ and $\bar{\Sigma}$ commute, and $\phi$ and $\Sigma$ cannot anticommute. So $\phi$ and $\Sigma$ commute. Thus $\Sigma \in C D C(\sigma)$, and we showed above there is a scalar $\lambda$ such that the fixed space of $\lambda \cdot \Sigma$ contains $H$. Now since $\Sigma \in C D C(\sigma)$, we have $\Sigma \in C D C\left({ }^{\vee}\right)$ since the contragredient mapping is an isomorphism of $\mathrm{GL}_{m}(W)$ onto $\mathrm{GL}_{m}\left(W^{\prime}\right)$ where $W^{\prime}$ denotes the dual space of $W$. Dualizing the above reasoning, we see that there is a scalar $\beta$ such that the residual space of $\beta \Sigma$ is contained in $L$. Since $m \geqq 3$, $\beta=\lambda$. Hence $\bar{\Sigma}=(\beta \Sigma)^{-} \in \bar{T}(L, H)$. Q.E.D.

COROLlary. With the hypotheses of $2.1, C D C(\bar{\sigma})$ is abelian.

Definition. An element of $\bar{\sigma}$ of $\operatorname{PGL}(W)$ is called a projective shearing if there is a shearing $\Sigma \in \mathrm{GL}(W)$ such that $\bar{\Sigma}=\bar{\sigma}$.

2.2. Let $n \geqq 3$ and $G_{1}$ be any subgroup of $U_{n}(V)$ that has enough projective transvections. Let $\bar{\sigma}_{1}$ and $\bar{\sigma}_{2}$ be nontrivial projective shearings in $G_{1}$. Then $C\left(\bar{\sigma}_{1}\right)=C\left(\bar{\sigma}_{2}\right)$ iff $L_{1}=L_{2}$ where $L_{i}$ is the proper line of $\bar{\sigma}_{i}$.

Proof. Suppose $L_{1}=L_{2}$. Then it follows from the proof of 2.9 of [5] that $C\left(\sigma_{1}\right)=C\left(\sigma_{2}\right)$, and so $\left(C\left(\sigma_{1}\right)\right)^{-}=\left(C\left(\sigma_{2}\right)\right)^{-}$. Since nothing can anticommute with a shearing for $n \geqq 3$ we see $C\left(\bar{\sigma}_{1}\right)=C\left(\bar{\sigma}_{2}\right)$.

Conversely, suppose $C\left(\bar{\sigma}_{1}\right)=C\left(\bar{\sigma}_{2}\right)$. Since nothing can anticommute with a shearing, this implies $C\left(\sigma_{1}\right)=C\left(\sigma_{2}\right)$ where the last two centralizers are taken in the group $\Delta_{1}$. Suppose $L_{1} \neq L_{2}$. Then the hyperplanes $L_{1}^{*}$ and $L_{2}^{*}$ are distinct so there is an isotropic line $L$ which is in $L_{1}^{*}$ but not in $L_{2}^{*}$. Let $\sigma_{3}$ be a nontrivial transvection in $\Delta_{1}$ with proper line $L$. Then $\sigma_{3} \in C\left(\sigma_{1}\right)$ but $\sigma_{3} \notin C\left(\sigma_{2}\right)$, which contradicts $C\left(\sigma_{1}\right)=C\left(\sigma_{2}\right)$. Hence $L_{1}=L_{2}$.

Q.E.D.

2.3. Let $m \geqq 4$ and let $\Lambda$ be an isomorphism of $G$ onto $G_{1}, \Lambda: G \rightarrow G_{1}$. Let $\bar{\sigma}$ be a projective transvection in $G$ with proper line $L$ and proper hyperplane $H$. Then $\Lambda \bar{\sigma}$ is a projective shearing.

Proof. Let $\bar{\Sigma}=\Lambda \bar{\sigma}$; we can assume $\bar{\sigma} \neq \overline{1}$ so $\bar{\Sigma} \neq \overline{1}$. Hence there is an isotropic line $L_{1}=F_{1} a$ such that $\Sigma L_{1} \neq L_{1}$. Let $T=\tau_{a, \lambda}$ be a nontrivial 
transvection in $\Delta_{1}$ with proper line $L_{1}$. By 1.1 we can assume $\Sigma$ and $T \Sigma^{-1} T^{-1}$ do not commute; a dimension argument shows they can not anticommute. Put $h=\Sigma T \Sigma^{-1} T^{-1}$, put $\Lambda \bar{\tau}=\bar{T}$ and set $f=\sigma \tau \sigma^{-1} \tau^{-1}$. Clearly $\Lambda \bar{f}=h$; and since $\bar{\Sigma}$ and $\bar{T} \bar{\Sigma}^{-1} \bar{T}^{-1}$ do not commute, $\bar{\sigma}$ and $\bar{\tau} \bar{\sigma}^{-1} \bar{\tau}^{-1}$ do not commute. Thus $\sigma$ and $\tau \sigma^{-1} \tau^{-1}$ do not commute.

Now $h=\left(\Sigma T \Sigma^{-1}\right) T^{-1}$ is the product of two transvections with distinct proper lines, $\Sigma L_{1}$ and $L_{1}$ respectively. Hence $h$ has residual space the plane $R=L_{1}+\Sigma L_{1}$. So $R$ is either a totally degenerate plane or regular plane. Now $\Sigma T \Sigma^{-1}=\tau_{\Sigma a, \lambda}$ and $T^{-1}=\tau_{a,-\lambda}$. From these formulae it follows $\Sigma T^{\prime} \Sigma^{-1}$ and $T^{-1}$ both act on $R=F_{1} a+\Sigma F_{1} a$. Thus either $\Sigma T \Sigma^{-1}$ and $T^{-1}$ both induce $1_{R}$, or they induce nontrivial transvections on $R$ with distinct proper lines. (Depending on whether $(a, \Sigma a)=0$ or $(a, \Sigma a) \neq 0$.) In any case $h \mid R \neq-1_{R}$ since $\chi(F) \neq 2$. By 1.7 of [5], $h^{2} \neq 1_{V}$ and surely $h^{2} \neq \gamma \cdot 1_{V}$, with $\gamma \neq 1$. So $\bar{h}^{2} \neq \overline{1}_{V}$; thus $\bar{f}^{2} \neq \overline{1}_{V}$ and so $f^{2} \neq 1_{V}$.

Now let us compute the fixed and residual spaces $R_{2}$ and $P_{2}$ of $f$. We know $R_{2} \subseteq L+\tau L$ and $P_{2} \supseteq H \cap \tau H$ by 1.1 of [5]. We will show $\tau H \neq H$, $\tau L \neq L, R_{2}=L+\tau L, P_{2}=H \cap \tau H, R_{2} \notin P_{2}$. We have shown above $\sigma$ and $\tau \sigma^{-1} \tau^{-1}$ do not commute. Their spaces are $L \subseteq H$ and $\tau L \subseteq \tau H$ respectively. Hence, $L \nsubseteq \tau H$ or $\tau L \nsubseteq H$ by 1.6 of [5]. So in particular $\tau L \neq L$ and $\tau H \neq H$. So $V=\tau H+H$ and so $R_{2}=L+\tau L$ by 1.2 of [5]. It follows that $P_{2}=\tau H \cap H$. Finally $R_{2}=L+\tau L \nsubseteq H \cap \tau H=P_{2}$. We have now shown $f$ satisfies the hypotheses of 1.1 of [8]. Hence by 1.1 of [8], $C D C(\bar{f})$ contains every element $\bar{\sigma}$ of $G$ such that the residual space of $\sigma$ is contained in $R_{2}$ and such that the fixed space of $\sigma$ contains $P_{2}$. From this it follows both $\bar{\sigma}$ and $\bar{\tau} \bar{\sigma}^{-1} \bar{\tau}^{-1}$ are in $C D C(\bar{f})$, and since these latter two elements do not commute, it follows $C D C(\bar{k})$ is nonabelian. Since $C D C(\bar{f})$ is nonabelian, $C D C(\Lambda \bar{f})=C D C(\bar{f})$ is nonabelian and so by $1.5, R$ is a regular plane, and therefore a hyperbolic plane.

Thus $\Lambda \bar{\sigma} \in C D C(\Lambda \bar{f})=C D C(\bar{h})$ since $\bar{\sigma} \in C D C(\bar{f})$. So by $1.3, \bar{\Sigma}=$ $\Lambda \bar{\sigma} \in C D C(\bar{h}) \subseteq(E(R))^{--}$where $R$ is the residual space of $h$. Hence we may assume $\Sigma$ has residual space a line or has residual space the hyperbolic plane $R$. If $R$ is the residual space of $\Sigma$ and $\Sigma \mid R$ is a scalar, then since $C D C(\bar{h}) \subseteq(E(R))^{-}$we see $\bar{\Sigma}$ centralizes $C D C(\bar{h})$ which contradicts the fact $\bar{\sigma} \notin C C D C(\bar{f})$; if $R$ is the residual space of $\Sigma$ and $\Sigma \mid R$ is not a scalar, then 1.2 shows $E(R) \subseteq C D C(\Sigma)$ contradicting the corollary of 2.1 that $C D C(\bar{\sigma})$ is abelian. So $\Sigma$ has residual space a line. Q.E.D.

Application to congruence groups. We now apply Theorem 2.3 to demonstrate the nonisomorphism, under appropriate hypotheses, of the linear, symplectic, and unitary congruence groups. The definition of these congruence groups will be taken as in [10]. 
2.4. Let $S$ be a linear congruence group whose associated vector space $W$ has dimension at least 4 . Let $S_{1}$ be a symplectic or a unitary congruence group whose associated form has Witt index at least 3 and whose associated field is of characteristic not 2. Then $S$ and $S_{1}$ are not isomorphic.

Proof. Let us define $G=S$ and define $G_{1}=S_{1}$. Now let us suppose that $\Lambda: G \rightarrow G_{1}$ is an isomorphism. Fix a line $L$ in $W$ and take two nontrivial projective transvections $\bar{\tau}$ and $\bar{\sigma}$ in $G$ with proper spaces $L \subset H$ and $L \subset H_{1}$ respectively, where $H$ and $H_{1}$ are two distinct hyperplanes of $W$. Then $C(\bar{\tau}) \neq C(\bar{\sigma})$ by 2.9 of [5]. However the composition $\bar{\tau} \bar{\sigma}$ is a nontrivial projective transvection in $G$, so $\Lambda \bar{\tau} \bar{\sigma}=\Lambda \bar{\tau} \Lambda \bar{\sigma}$ is a transvection or a quasisymmetry. However $C(\Lambda \bar{\tau}) \neq C(\Lambda \bar{\sigma})$ since $C(\bar{\tau}) \neq C(\bar{\sigma})$. But by the previous theorem $\Lambda \bar{\tau}$ and $\Lambda \bar{\sigma}$ are projective shearings; let them have residual lines $L_{1}$ and $L_{2}$ respectively. Then $L_{1} \neq L_{2}$ for if $L_{1}=L_{2}, 2.2$ would imply $C(\Lambda \bar{\tau})=C(\Lambda \bar{\sigma})$. Since $L_{1} \neq L_{2}$ the composition $\Lambda \bar{\tau} \Lambda \bar{\sigma}$ is neither a projective transvection nor a projective quasi-symmetry, which is a contradiction.

From the fact the groups $G$ and $G_{1}$ are not isomorphic, it follows the groups $S$ and $S_{1}$ cannot be isomorphic. For any isomorphism between $S$ and $S_{1}$ would induce in a natural way an isomorphism between $G$ and $G_{1}$. Q.E.D.

\section{REFERENCES}

1. H. Bass, J. Milnor and J.-P. Serre, Solution of the congruence subgroup problem for $\mathrm{SL}_{n}(n \geqq 3)$ and $\mathrm{Sp}_{2 n}(n \geqq 2)$, Inst. Hautes Études Sci. Publ. Math. No. 33 (1967), 59-137. MR 39 \#5574.

2. J. Dieudonné, La géométrie des groupes classiques, 2ième éd., Springer-Verlag, Berlin, 1963. MR 28 \#1239.

3. - On the automorphisms of the classical groups, Mem. Amer. Math. Soc. No. 2 (1951). MR 13, 531.

4. O. T. O'Meara and H. Zassenhaus, The automorphisms of the linear congruence groups over Dedekind domains, J. Number Theory 1 (1969), 211-221. MR 39 \#4292.

5. O. T. O'Meara, Group-theoretic characterization of transvections using CDC, Math. Z. 110 (1969), 385-394. MR 40 \#1486.

6. C. E. Rickart, Isomorphic groups of linear transformations, Amer. J. Math. 72 (1950), 451-464. MR 11, 729.

7. - Isomorphic groups of linear transformations. II, Amer. J. Math. 73 (1951), 697-716. MR 13, 532.

8. R. E. Solazzi, The automorphisms of certain subgroups of $\mathrm{PGL}_{n}(V)$, Illinois $\mathrm{J}$. Math. 16 (1972), 330-349.

9. - The automorphisms of the unitary groups and their congruence subgroups, Illinois J. Math. (to appear).

10. Isomorphism theory of congruence groups, Bull. Amer. Math. Soc. 77 (1971), 164-168.

Department of Mathematics, Indiana University, Bloomington, Indiana 47401 